\title{
Intracranial Stimulation Therapy for Epilepsy
}

\author{
Tara L. Skarpaas* and Martha J. Morrell* ${ }^{\dagger}$ \\ *NeuroPace, Inc., Mountain View, California 94043, †epartment of Neurology, Stanford University Medical Center, \\ Stanford 94043, California
}

Summary: Epilepsy is a common chronic neurological disorder effecting 1 to $2 \%$ of the population. Despite advances in anti-epileptic drug therapy, epilepsy surgery, and vagus nerve stimulation, approximately $30 \%$ of patients continue to have seizures. Intracranial stimulation is currently under investigation as an adjunctive treatment to anti-epileptic medications in adults with medically intractable epilepsy. Several different approaches are now being investigated. Specifically, acute and long-term clinical studies have delivered stimulation either to inhibitory regions outside the seizure focus or directly to the seizure focus. These studies have demonstrated the safety of intracranial stimulation and proof of principle in epilepsy patients. In addition to the different locations tested, clinical studies have also used different temporal patterns of stimulation. The majority of studies have used open-loop or scheduled stimulation, in which, stimulation is delivered on a fixed schedule and is independent of electrographic activity. In contrast, a number of recent investigations have demonstrated the feasibility of closed-loop or responsive stimulation, which is stimulation that is contingent upon the detection of epileptiform activity.

This chapter will review the acute and long-term clinical studies of intracranial stimulation, including focal, and nonfocal, and open-loop and responsive stimulation. We will also discuss the optimization and safety of therapeutic parameters used in neurostimulation for epilepsy. Key Words: Epilepsy, responsive, neurostimulation, device, open-loop, closed-loop.

\section{INTRODUCTION}

Approximately 2.5 million people in the United States have active epilepsy and 181,000 new cases of epilepsy are diagnosed each year. By 20 years of age, epilepsy has developed in $1 \%$ of the population, and by 75 years of age, the prevalence of epilepsy reaches 3\%. ${ }^{1}$ Despite advances in anti-epileptic drug (AED) therapy, epilepsy surgery, and vagus nerve stimulation (VNS), approximately $30 \%$ of patients continue to have seizures. ${ }^{2,3}$ Thus, alternative treatment strategies are needed.

\section{ACUTE CLINICAL STUDIES}

Intracranial stimulation is currently under investigation as an adjunctive treatment to anti-epileptic medications in adults with medically intractable epilepsy who are not candidates for potentially curative epilepsy surgery. As early as the 1950s, Penfield and Jasper ${ }^{4}$ observed that cortical stimulation could disrupt epileptiform activity. Extinction was observed at the primary electrodes immediately after deliv-

Address correspondence and reprint requests to: Martha J. Morrell, M.D., Clinical Professor of Neurology, Stanford University, NeuroPace, Inc., 1375 Shorebird Way, Mountain View, CA 94043. E-mail: mmorrell@neuropace.com. ery of stimulation, after an afterdischarge, or after a spontaneous epileptic discharge, whereas suppression of normal and epileptiform activity was observed at distant sites and over large areas. Extinction was thought to represent postexcitatory depression and suppression to result from activation of inhibitory pathways, suggesting that stimulation at the seizure focus, as well as stimulation of inhibitory regions outside the focus could be used to inhibit epileptiform activity.

Subsequently a number of acute studies of both focal and nonfocal intracranial stimulation have been conducted, primarily in patients implanted with intracranial electrodes for purposes of localizing the seizure focus. In these studies, two main approaches to the timing of stimulus delivery have been explored, namely open-loop and closed-loop stimulation. Open-loop stimulation is delivered on a scheduled basis and is not contingent on the presence of epileptiform activity. In contrast, closed-loop (or responsive) stimulation provides stimulation only when abnormal (ictal or interictal) discharges are detected.

Kinoshita et al.,,6 in 2004 and 2005, respectively, tested the effect of focal open-loop cortical stimulation on interictal activity and electrocorticogram (ECoG) power spectra during extra-operative monitoring and functional mapping in a total of five patients with epilepsy of frontal lobe or mesial temporal lobe origin. The 
spike frequency and ECoG power spectra before and after high $(50 \mathrm{~Hz})$ and low $(0.9 \mathrm{~Hz})$ frequency stimulation were compared. There was a significant reduction in the number of interictal spikes post-stimulation compared with a pre-stimulation baseline. In addition, high frequency stimulation significantly decreased the power of low voltage fast activity. Other groups have also demonstrated a significant reduction in interictal spiking after open-loop stimulation of the hippocampus ${ }^{7,8}$ and motor cortex. ${ }^{9}$

Kinoshita et al., ${ }^{6} 2005$ also examined the effect on epileptiform activity of $50 \mathrm{~Hz}$ stimulation provided by cortical electrodes distant from the seizure focus (nonfocal). This nonfocal stimulation did not inhibit spiking and did not alter the power of high-frequency activity. However, the regions stimulated in this study were different from nonfocal regions previously reported to have a suppressive effect on epileptic discharges. For instance, Zumsteg et al., ${ }^{10} 2006$ demonstrated that open-loop stimulation of the anterior thalamic nucleus produced inhibition in the hippocampus. Moreover several studies have reported an effect of nonfocal stimulation on epileptiform activity and clinical seizures after stimulation of regions thought to be associated with the activation of inhibitory networks including the cerebellum, ${ }^{11}$ caudate nucleus, ${ }^{12}$ and several thalamic nuclei. ${ }^{13}$

A number of studies have also examined the effect of focal and nonfocal closed-loop stimulation on epileptiform activity in patients with subdural electrodes placed for pre-surgical evaluation. Lesser et al., ${ }^{14} 1999$ examined the effect of cortical stimulation in 17 patients undergoing functional mapping for language, motor, and sensory regions. Afterdischarges produced by a localizing stimulus during the mapping could be aborted by a brief burst of stimulation delivered via the same electrodes used to elicit the afterdischarge. These results were extended by Motamedi et al., ${ }^{15} 2002$ who demonstrated that the success of a brief burst to abort an afterdischarge was enhanced if the stimulus was applied early to the primary electrodes and at a specific phase of the afterdischarge.

Trials of closed-loop stimulation have been conducted in patients undergoing evaluation with intracranial electrodes as part of an epilepsy surgery evaluation. Peters et al., ${ }^{16} 2001$ described a nonimplantable bedside prototype that included an ECoG acquisition system and computers that were used to store, process, and analyze ECoGs, and to control stimulation delivered through subdural electrodes by a Grass S12 stimulator (Grass Technologies, Warwick, RI). This system was used to deliver closed-loop stimulation in response to spontaneous seizures in eight patients. ${ }^{17}$ Seizures originating from a discrete location received stimulation to the seizure onset zone. In contrast, seizures originating from multiple independent foci were treated with stimulating electrodes implanted in the anterior thalamic nucleus. All patients received high fre- quency $(100-500 \mathrm{~Hz})$ biphasic stimulation. Closed-loop stimulation reduced the seizure rate by $55.5 \%$ in the focal group and $40.8 \%$ in the nonfocal group. This study demonstrated that automated closed-loop, high-frequency stimulation could be delivered in close temporal proximity to the seizure onset and suggested that closed-loop stimulation might produce a significant reduction in clinical seizures.

Results from a small subset of patients enrolled in a large multi-center acute study of a second bedside prototype also suggested that closed-loop stimulation could alter or block electrographic seizures. ${ }^{18}$ The external responsive neurostimulator (eRNS) is a battery-operated device that processes digital data in real-time and has three different detection tools that can be programmed to identify a patient's electrographic seizure onset. ${ }^{18}$ The authors reported that responsive stimulation terminated some electrographic seizures. Figure 1 shows examples of electrographic seizures in one patient (FIG. 1A) without and (FIG. 1C) with closedloop stimulation. Note the return to baseline electrographic activity after stimulation in Figure 1C.

These acute studies suggest that both focal and nonfocal stimulation can acutely inhibit epileptiform activity and that both open-loop and closed-loop stimulation can be effective. However, the acute studies do not address the effect of stimulation on clinical seizures, nor do they address the efficacy or safety of long-term intracranial stimulation.

\section{CHRONIC CLINICAL STUDIES AND IMPLANTABLE DEVICES}

The first long-term clinical studies of chronic brain stimulation provided open-loop cerebellar stimulation and reported a significant reduction in clinical and electrographic seizures. ${ }^{11}$ However, subsequent randomized trials did not show benefit. ${ }^{19}$ Other studies of brain stimulation in persons with medically uncontrolled epilepsy have shown a reduction in seizure frequency after stimulation of regions outside the seizure focus including several thalamic nuclei, subthalamic nucleus, caudate, mammillary bodies, locus coeruleus, and basal ganglia. Most results indicate that indirect stimulation can more successfully inhibit secondarily generalized seizures than complex partial seizures. ${ }^{20}$

To date, a number of small clinical studies have tested the efficacy of implantable devices that deliver stimulation to the seizure onset zone. Many of these studies have focused on open-loop stimulation to mesial temporal structures. Velasco et al., ${ }^{8} 2000$ treated patients implanted with hippocampal depth leads (placed via an occipital approach) with high-frequency biphasic stimulation. Clinical seizures and interictal spikes were blocked with $130 \mathrm{~Hz}, 200$ to $400 \mu \mathrm{A}$, stimulation. This stimulation produced no histopathological tissue damage and no negative effect on short-term memory. In addi- 


\section{A Stored ECoG Record from eRNS}

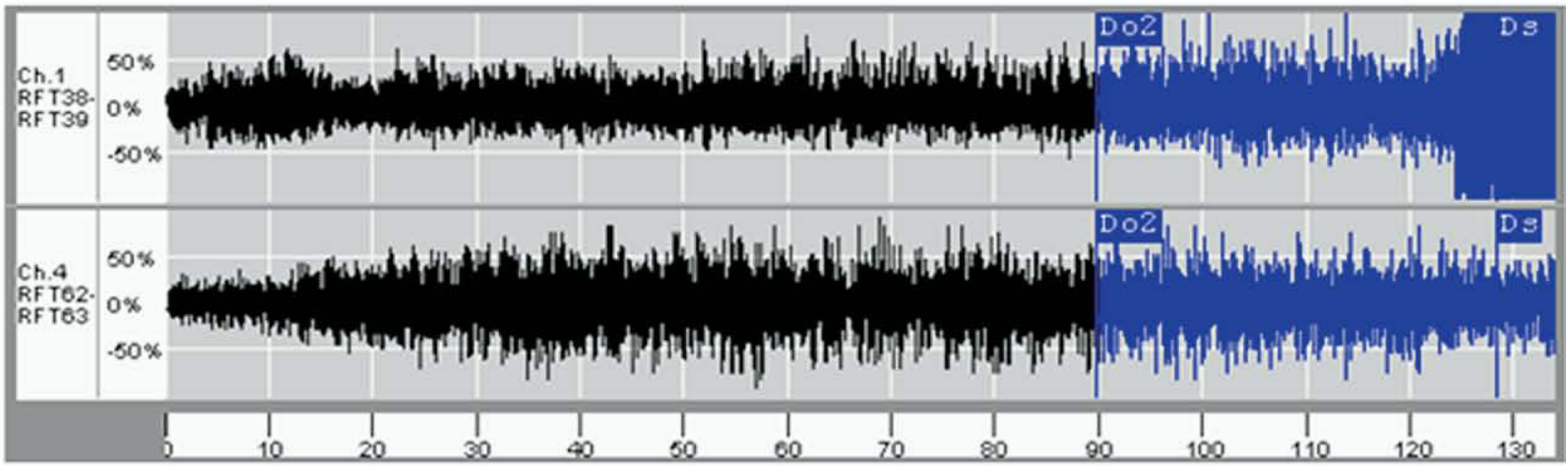

B

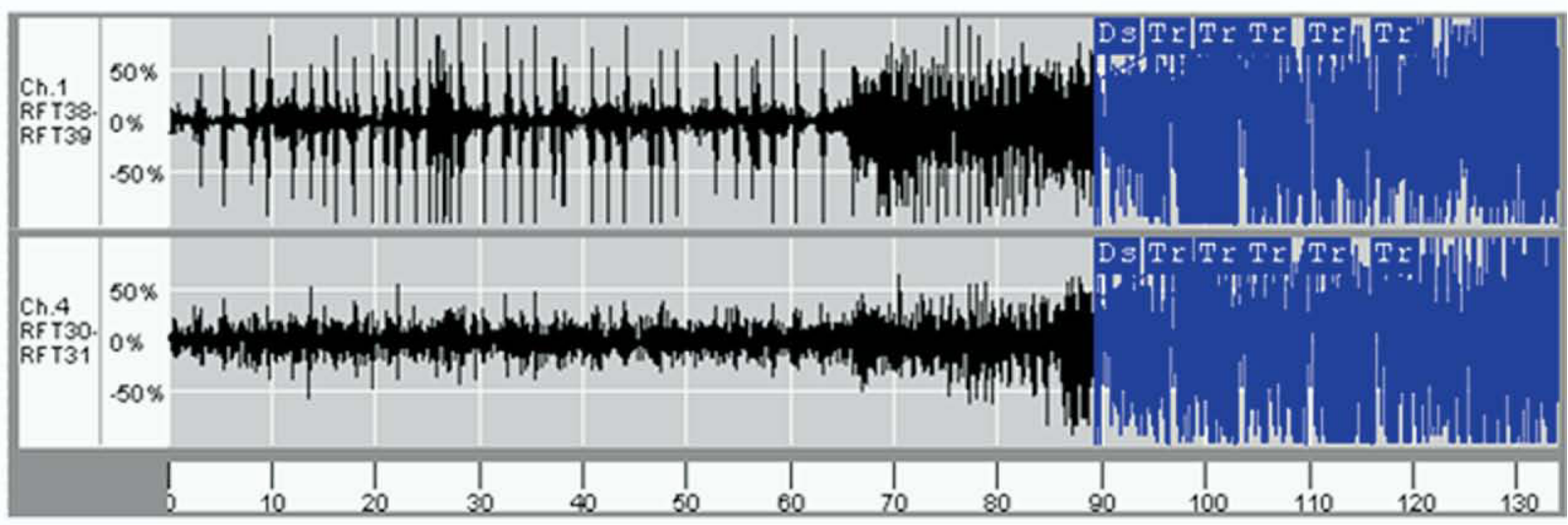

C

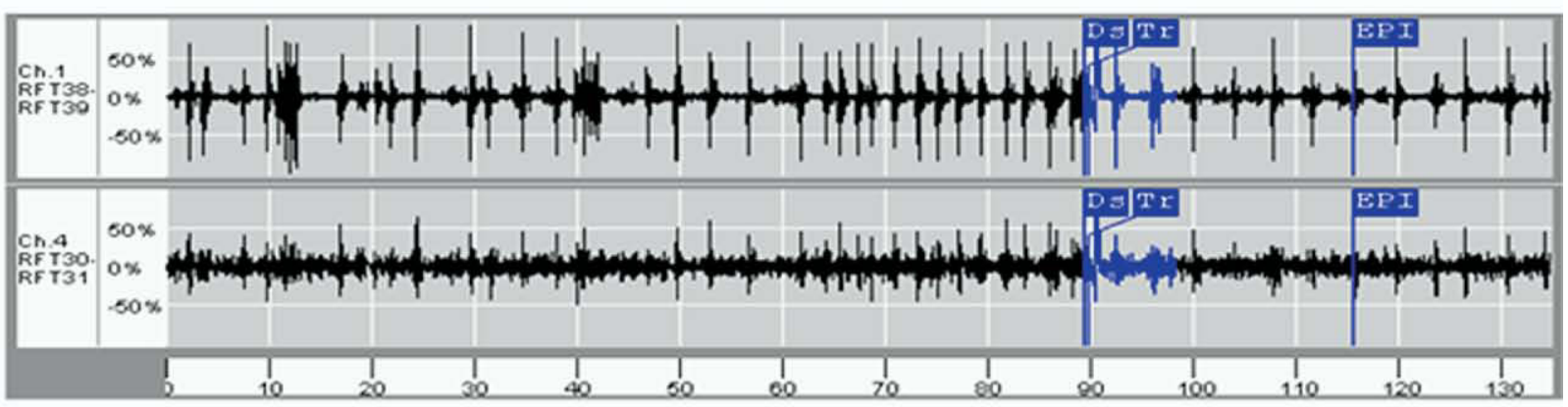

\section{Close-up of ECoG at Time of Responsive Stimulation}

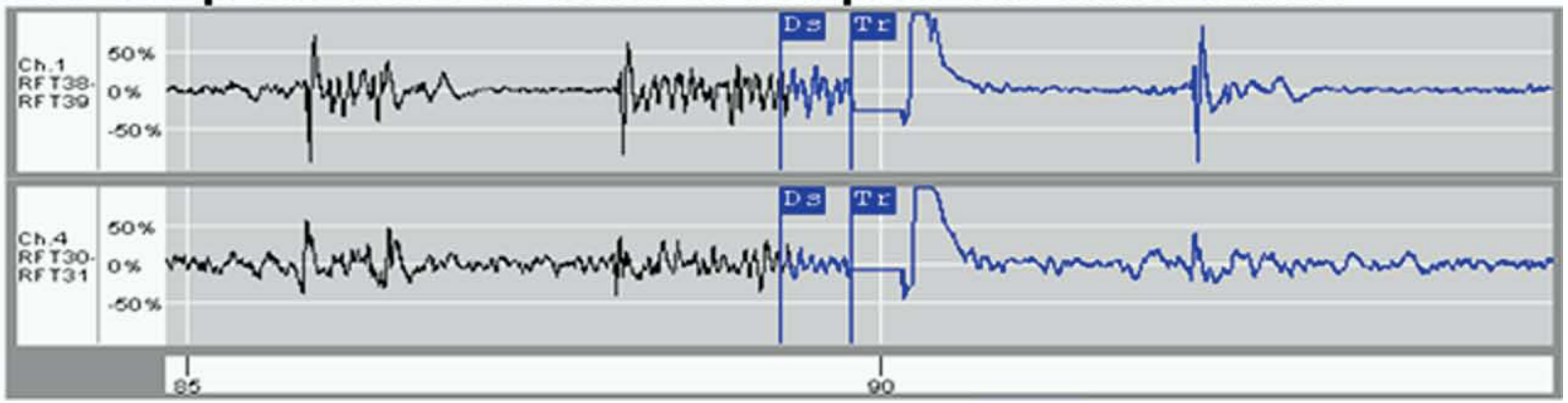

FIG. 1. Electrocorticographic (ECoG) recordings of electrographic seizures from the same patient. (A) Seizure recorded without stimulation. The electrographic activity correlated with a clinically reported seizure. Detection, denoted by blue, had not been tuned for the seizure onset and was late. (B) Seizure recorded with focal high-frequency stimulation delivered by the eRNS late after the seizure onset. Stimulation is denoted by Tr. Again detection had not been tuned for the seizure onset and thus detection and stimulation were late and the seizure progressed. (C) Early detection of electrographic seizure onset pattern and early focal responsive high-frequency stimulation delivered to the seizure onset by the eRNS. FFT = fast Fourier transform. 
tion, they noted that stimulation therapy was most effective when the electrodes extended to the anterior pes hippocampus near the amygdala. A subsequent study followed patients for 5 to 21 months after implantation of amygdala hippocampal depth leads and a pulse generator. $^{21}$ They noted a $>50 \%$ reduction in seizure frequency in 4 of 7 patients, one of whom was seizure free for 1.5 years. In addition, 2 of the patients could be tapered off of one AED. No side effects related to the stimulation were reported. Although one randomized trial of hippocampal stimulation demonstrated a smaller nonsignificant seizure reduction, ${ }^{22}$ a recent double-blind trial in 9 patients demonstrated a significant reduction of seizure frequency in response to hippocampal stimulation. ${ }^{23} \mathrm{Im}$ portantly, there were no adverse effects reported in either study and the stimulation did not have any detrimental effects on cognitive and memory functions.

Focal stimulation of neocortical onsets has also been used to treat intractable seizures. One patient had a seizure focus in the primary motor hand region. ${ }^{9}$ During functional brain mapping, it was noted that direct $50 \mathrm{~Hz}$ stimulation for 3 minutes at $2 \mathrm{mV}$ significantly suppressed interictal spiking for 10 minutes. The spiking then gradually returned but could be suppressed with subsequent stimulation. The patient was implanted with a permanently indwelling electrode array at the seizure focus and a pulse generator was placed in the subclavicular area. The patient received cyclical open-loop stimulation (biphasic $50 \mathrm{~Hz}, 2.1 \mathrm{mV}$ for 3 min on, 10 min off) for 5 years without evidence of injury. Moreover, the stimulation reduced the seizure frequency by more than $90 \%$.

The first implantable responsive neurostimulator for epilepsy, the NeuroPace RNS System (NeuroPace, Inc., Mountain View, CA), is currently being evaluated for safety and efficacy in clinical trials for the treatment of intractable partial onset epilepsy in adults. A schematic of the implanted device appears in Figure 2. The implantable components include a cranially implanted neurostimulator that is connected to up to two depth and/or strip leads containing four electrodes each. An external programmer is used by the physician to program detection and stimulation parameters and retrieve stored electrographic activity. A wand allows wireless communication between the stimulator and the programmer. A data transmitter is provided to the patient to allow uploading and remote monitoring of device data between clinic visits. The RNS System continually analyzes the patient's electrographic activity and automatically delivers electrical stimulation to the seizure focus when the patient's characteristic epileptiform activity is detected. An initial single-center report demonstrated a $45 \%$ decrease in seizures in 7 of 8 patients with a mean follow-up of 9 months. $^{24}$ A randomized, double-blind, sham-controlled pivotal clinical trial is currently being conducted to demonstrate the safety and efficacy of the RNS System.

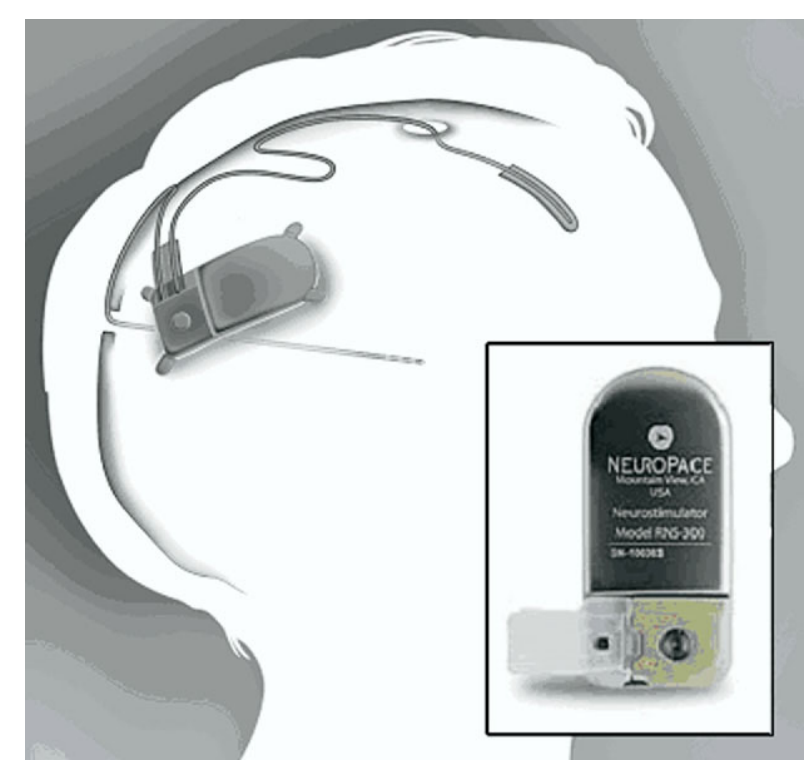

FIG. 2. Schematic of the NeuroPace RNS neurostimulator, depth and strip leads. Inset shows RNS neurostimulator.

\section{OPTIMIZING THERAPEUTIC STIMULUS PARAMETERS}

A variety of parameters may influence the response to both open- and closed-loop stimulation. These parameters include dose or number of stimulations, stimulus frequency, current intensity, stimulus duration, stimulus waveform, and the location. Both high and low frequencies have been shown to inhibit epileptiform activity and seizures, and the optimal stimulus frequency may depend on the region being stimulated. ${ }^{12}$ In addition, the efficacy of responsive stimulation may be influenced by how early the electrographic seizure can be detected. For instance, stimulations delivered later in the seizure failed to terminate the electrographic event, whereas stimulations delivered early resulted in a return to baseline activity (FIG. 3). Thus adjusting detection parameters to detect at the earliest sign of an electrographic event may also be important in optimizing responsive stimulation.

\section{Safety of intracranial stimulation}

No damage has been observed in human cortex exposed to intermittent stimulation used to perform functional mapping as long as the charge delivered remains below 50 to $60 \mu \mathrm{C} / \mathrm{cm}^{2} /$ phase..$^{25,26}$ However, there is a safety concern that chronic subthreshold stimulation, such as that used by implantable devices for the treatment of epilepsy, may induce neural injury. Experience with deep brain stimulation for Parkinson's disease suggests that long-term continuous stimulation can be delivered safely. ${ }^{27}$ Animal studies have suggested that tissue damage due to stimulation is correlated with charge density per phase, and the total charge per phase, as well as with cumulative exposure of tissue to stimulation. ${ }^{28}$ Intermittent stimulation provides less cumulative expo- 
A
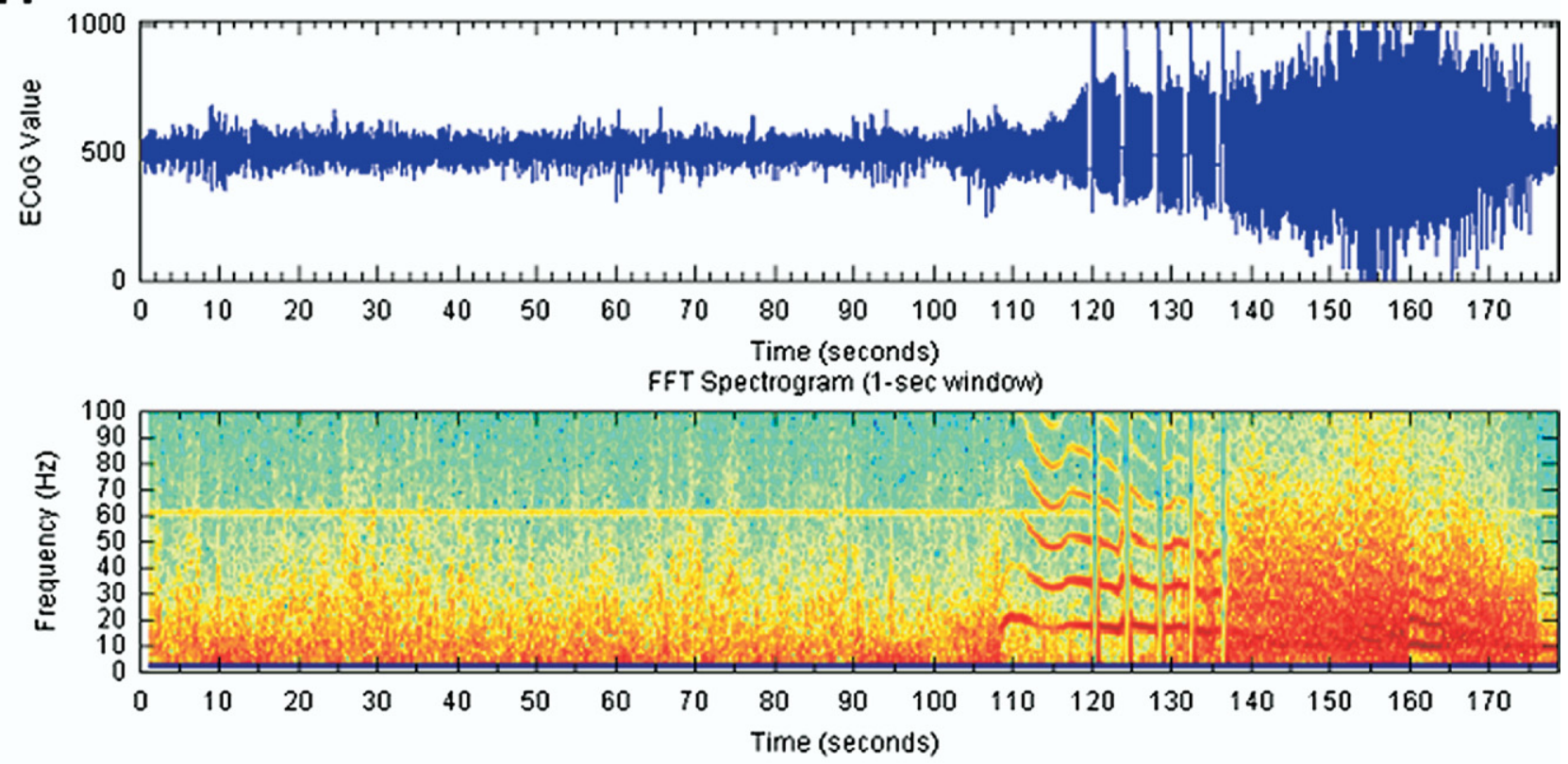

B
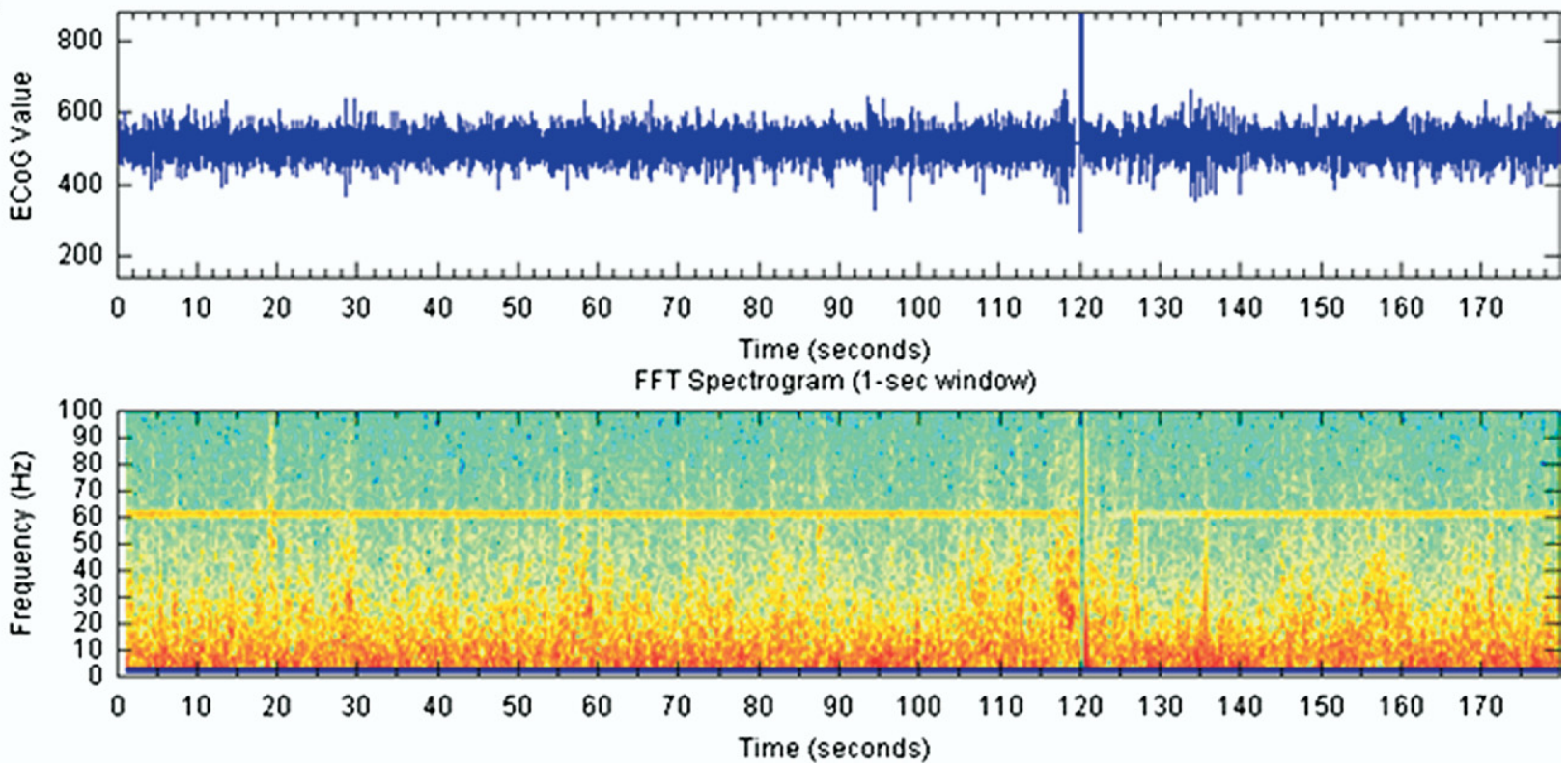

FIG. 3. Time series and spectrograms of electrographic seizures receiving $(A)$ late stimulation and (B) early stimulation relative to the seizure onset using the implantable RNS System. Note that (A) the seizure continues to evolve when focal stimulation is delivered several seconds after the onset of the seizure, whereas (B) the electrographic activity does not evolve when focal stimulation is delivered earlier.

sure than continuous stimulation. Further studies are required to determine if either open- or closed-loop stimulation produces damage to tissue after long-term use.

Surgically implanting devices to deliver intracranial stimulation for the treatment of epilepsy may be associated with some additional risks. Reports from the implantation of deep brain stimulators for Parkinson's disease, pain, and hearing loss suggest that the procedure is associated with a $5 \%$ risk of infection and a 5 to $7.5 \%$ risk of intracerebral hemorrhage. ${ }^{20}$ Surgical risks may vary depending on the site of implantation, the size of the device, and the general health of the patient population.

\section{SUMMARY}

Implantable neurostimulation devices may provide a new therapy for medically intractable epilepsy patients. One advantage of intracranial stimulation for the treatment of epilepsy is that the therapy is largely reversible. In addition, stimulation therapy can be delivered to a 
specific onset region or inhibitory network and can be adjusted for individual patients. Thus, intracranial stimulation may offer more specificity than anti-epileptic drugs. Ongoing clinical trials of intracranial stimulation may reveal that that there is more than one approach to brain stimulation that is safe and effective. Further studies will be required to identify patients who might benefit from stimulation, to identify safe and effective stimulation targets and ascertain the optimal stimulation parameters for individuals or groups of patients.

\section{REFERENCES}

1. Begley CE, Famulari M, Annegers JF, et al. The cost of epilepsy in the United States: an estimate from population-based clinical and survey data. Epilepsia 2000;4:342-351.

2. Brodie MJ, Dichter MA. Antiepileptic drugs. N Engl J Med 1996; 334:168-175.

3. Morrell MJ. Antiepileptic medications for the treatment of epilepsy. Semin Neurol 2002;22:247-258.

4. Penfield W, Jasper H. Electrocorticography. In: Little, ed. Epilepsy and the functional anatomy of the human brain. Boston: Brown; 1954.

5. Kinoshita M, Ikeda A, Matsumoto R, et al. Electric stimulation on human cortex suppresses fast cortical activity and epileptic spikes. Epilepsia 2004;45:787-791.

6. Kinoshita M, Ikeda A, Matsuhashi M, et al. Electric cortical stimulation suppresses epileptic and background activities in neocortical epilepsy and mesial temporal lobe epilepsy. Clin Neurophysiol 2005;116:1291-1299.

7. Boon P, Vonck K, De Herdt V, et al. Deep brain stimulation in patients with refractory temporal lobe epilepsy. Epilepsia 2007;48: $1551-1560$

8. Velasco A, Velasco M, Velasco F, et al. Subacute and chronic electrical stimulation of the hippocampus on intractable temporal lobe seizures: preliminary report. Arch or Med Res 2000;31:316328.

9. Elisevich K, Jenrow K, Schuh L, Smith B. Long-term electrical stimulation-induced inhibition of partial epilepsy. Case report. J Neurosurg 2006;105:894-897.

10. Zumsteg D, Lozano A, Wennberg R. Mesial temporal inhibition in a patient with deep brain stimulation of the anterior thalamus for epilepsy. Epilepsia 2006;47:1958-1962.

11. Cooper I, Upton A. Use of chronic cerebellar stimulation for disorders of disinhibition. Lancet 1978;1:595-600.

12. Sramka M, Chkhenkeli SA. Clinical experience in intraoperational determination of brain inhibitory structures and application of implanted neurostimulators in epilepsy. Stereotact Funct Neurosurg 1990;54-55:56-59.
13. Upton AR, Cooper IS, Springman M, Amin I. Suppression of seizures and psychosis of limbic system origin by chronic stimulation of anterior nucleus of the thalamus. Int J Neurol 1985;1920:223-230.

14. Lesser RP, Kim SH, Beyderman L, et al. Brief bursts of pulse stimulation terminate afterdischarges caused by cortical stimulation. Neurology 1999;53:2073-2081.

15. Motamedi GK, Lesser RP, Miglioretti DL, et al. Optimizing parameters for terminating cortical after discharges with pulse stimulation. Epilepsia 2002;43:836-846.

16. Peters T, Bhavaraju N, Frei M, Osorio I. Network system for automated seizure detection and contingent delivery of therapy. J Clin Neurophysiol 2001;18:545-549.

17. Osorio I, Frei MG, Sunderam S, et al. Automated seizure abatement in humans using electrical stimulation. Ann Neurol 2005;57: $258-268$.

18. Kossoff EH, Ritzl EK, Politsky JM, et al. Effect of an external responsive neurostimulator on seizures and electrographic discharges during subdural electrode monitoring. Epilepsia 2004;45: $1560-1567$.

19. Oommen J, Morrell M, Fisher RS. Experimental electrical stimulation therapy for epilepsy. Curr Treat Options Neurol 2005;7:261271.

20. Kerrigan J, Litt B, Fisher R, et al. Electrical stimulation of the anterior nucleus of the thalamus for the treatment of intractable epilepsy. Epilepsia 2004;45:346-354.

21. Vonck K, Boon P, Claeys P, Dedeurwaerdere S, Achten R, Van Roost D. Long-term deep brain stimulation for refractory temporal lobe epilepsy. Epilepsia 2005;46(suppl 5):98-99.

22. Tellez-Zenteno J, McLachlan R, Parrent A, Kubu C, Wiebe S. Hippocampal electrical stimulation in mesial temporal lobe epilepsy. Neurology 2006;66:1-5.

23. Velasco AL, Velasco F, Velasco M, Trejo D, Castro G, CarrilloRuiz JD. Electrical stimulation of the hippocampal epileptic foci for seizure control: a double-blind, long-term follow-up study. Epilepsia 2007;48:1895-1903.

24. Fountas K, Smith J, Murro A, Politsky J, Park Y, Jenkins P. Implantation of a closed-loop stimulation in the management of medically refractory focal epilepsy. Stereotactic and Funct Neurosurg 2005;83:153-158.

25. Gordon B, Lesser R, Rance N, et al. Parameters for direct cortical electrical stimulation in the human: histopathologic confirmation. Electroenchepalog and Clin Neurophysiol 1990;75:371-377.

26. Risinger M, Gumnit R. Intracranial electrophysiologic studies. Neuroimaging and Clin N Am 1995;5:559-573.

27. Haberler C, Alesch F, Mazal PR, et al. No tissue damage by chronic deep brain stimulation in Parkinson's disease. Ann Neurol 2000;48:372-376.

28. Yuen TG, Agnew WF, Bullara LA, Jacques S, McCreery DB. Histological evaluation of neural damage from electrical stimulation: considerations for the selection of parameters for clinical application. Neurosurgery 1981;9:292-299. 